\title{
Bifurcation in Granular Materials:
}

\section{A Multiscale Approach}

\author{
François NICOT, Luc SIBILLE and Félix DARVE
}

\subsection{Introduction}

The notion of failure can be encountered in many fields, irrespective of the scale considered. This notion is essential in materials sciences where failure can be investigated on the specimen (the material point) scale. It is also meaningful in civil engineering to prevent or to predict the occurrence of failure on a large scale.

For geomaterials, known as non-associate materials, several failure modes can be encountered strictly within the plastic surface. From a mathematical point of view, this feature is essentially related to the non-symmetry of the tangent constitutive tensor. Whereas the localized mode describes a failure corresponding to a discontinuous displacement field, the diffuse mode is associated with a homogenous kinematic field with no localization pattern. It has been shown that this failure mode can be predicted by the vanishing of the second-order work [NIC 07a and b]. Introduced by Hill [HIL 58], this quantity (hereafter denoted by $W_{2}$ ) is defined from

the inner product of the incremental first Piola-Kirchoff stress tensor $(\overline{\bar{\Pi}})$ with the incremental displacement gradient tensor:

$$
W_{2}=\int_{V_{o}} \delta \Pi_{i j}\left(\frac{\partial\left(\delta u_{i}\right)}{\partial X_{j}}\right) d V_{o}
$$


The interest of this semi-Lagrangian formulation lies in that all variables are reported to the fixed initial configuration defined by the volume $V_{o}$ and the coordinates $X_{i}$. For a material point corresponding to a representative volume element (RVE) of a granular material, equation [11.1] simplifies into the following expression:

$$
W_{2}=V_{o} \delta \Pi_{i j}\left(\frac{\partial\left(\delta u_{i}\right)}{\partial X_{j}}\right)
$$

The second-order work can also be expressed under an Eulerian formulation, introducing the Cauchy stress tensor $\overline{\bar{\sigma}}$ :

$$
W_{2}=V\left(\delta \overline{\bar{\sigma}}+\operatorname{div}(\delta \vec{u}) \overline{\bar{\sigma}}-\overline{\bar{\sigma}}(\overline{\bar{L}})^{t} \delta t\right): \overline{\bar{L}} \delta t
$$

where $L_{i j}$, such as $L_{i j} \delta t=\frac{\partial\left(\delta u_{i}\right)}{\partial x_{j}}$, is the general term of the velocity gradient tensor $\overline{\bar{L}}$. It follows that the second-order work is the combination of three terms: $V \delta \overline{\bar{\sigma}}: \overline{\bar{L}} \delta t$ is a material term, $\delta V \overline{\bar{\sigma}}: \overline{\bar{L}} \delta t$ is related to the change in volume, and $V \overline{\bar{\sigma}}(\overline{\bar{L}})^{t}: \overline{\bar{L}} \delta t^{2}$ is associated with the change in the texture [NIC 07a and c].

According to the small strain approximation, $\overline{\bar{D}} \delta t=\delta \bar{\varepsilon}$, where $\overline{\bar{D}}=\frac{1}{2}\left(\overline{\bar{L}}+\overline{\bar{L}}^{t}\right)$ denotes the strain rate tensor, and $\delta \overline{\bar{\varepsilon}}$ is the incremental small strain tensor. From the symmetry of this tensor, it follows that:

$$
W_{2}=V \delta \overline{\bar{\sigma}}: \delta \bar{\varepsilon}+\delta V \overline{\bar{\sigma}}: \delta \overline{\bar{\varepsilon}}-V \overline{\bar{\sigma}}: \overline{\bar{L}}^{2}(\delta t)^{2}
$$

The different formulations of the second-order work introduce macroscopic tensorial variables that represent both the complex force and displacement distributions within the granular specimen. The vanishing of the second-order work stems therefore from microstructural origins for which the local variables (contact forces and relative displacements between adjoining particles) are relevant. As a consequence, since the vanishing of the second-order work is a proper criterion for detecting the occurrence of a certain failure mode in geomaterials, it makes sense to track the microstructural origin of this macroscopic vanishing. This analysis will be 
first carried out by considering our micro-directional model [NIC 05], which is basically a micromechanically based constitutive relation. Then, this approach will be extended based on a general micromechanical derivation.

\subsection{Microstructural origin of the vanishing of the second-order work}

\subsubsection{The micro-directional model}

The micro-directional model is a multi-scale relation between the Cauchy stress tensor $d \overline{\bar{\sigma}}$ and the strain tensor $d \overline{\bar{\varepsilon}}$ by taking micro-mechanical characteristics into account. In this approach, the granular assembly is described as a distribution of contacts within adjoining particles. Each contact is associated with a given direction of the physical space, corresponding to the normal direction to the tangent contact plane. The texture is therefore described by the distribution of contacts along each direction of the physical space. The probability that some contacts exist in a given direction is investigated and local variables are averaged in each direction, so that directional variables are introduced. Fundamentally, this model is based on a homogenization procedure within a representative volume element (RVE) that can be resolved in the three following basic stages (for more details, see [NIC 05]).

The stress average corresponds to the Love formula ([LOV 27]; [WEB 66]; [CHR 81]; [MEH 82]):

$$
\sigma_{i j}=\frac{1}{V} \sum_{c=1}^{N_{c}} F_{i}^{c} l_{j}^{c}
$$

where $\overrightarrow{l^{c}}$ is the branch vector joining the centers of particles in contact on contact $c$, $\overrightarrow{F^{c}}$ is the contact force, and the sum is extended to all the $N_{c}$ contacts occurring in the RVE of volume $V$. The norm of the branch vector $\overrightarrow{l^{c}}$ is assumed to be a constant parameter (equal to the mean diameter of the grains) whose evolution over the loading programs is ignored. This ensures that the terms $\overrightarrow{F^{c}}$ and $\overrightarrow{l^{c}}$ are uncoupled. The discrete summation given in equation [11.5] can be replaced with a continuous integration over all the contact directions in the physical space. This scheme confers the directional character to the model:

$$
\sigma_{i j}=2 r_{g} \iint_{D} \hat{F}_{i} n_{j} \omega d \Omega
$$


where $\omega$ is the density of contacts along each space direction $\vec{n}, r_{g}$ denotes the mean radius of the sphere-shaped grains, $\hat{\vec{F}}$ is the average of all contact forces $\overrightarrow{F^{c}}$ associated with contacts oriented in the direction $\vec{n}$, and $d \Omega$ is the elementary solid angle. After differentiation it follows that:

$$
\delta \sigma_{i j}=2 r_{g} \iint_{D} \delta \hat{F}_{i} n_{j} \omega d \Omega+2 r_{g} \iint_{D} \hat{F}_{i} n_{j} \delta \omega d \Omega
$$

The kinematic projection relation is given by:

$$
\delta \hat{u}_{i}(\vec{n})=2 r_{g} \delta \varepsilon_{i j} n_{j}
$$

where $\hat{\vec{u}}(\vec{n})$ is the directional kinematic variable linked to $\hat{\vec{F}}(\vec{n})$ along the contact direction $\vec{n}$.

The local behavior is described by introducing a constitutive relationship between both average normal force $\hat{F}_{n}$ and tangential force $\hat{F}_{t}$ and both average relative normal displacement $\hat{u}_{n}$ and tangential displacement $\hat{u}_{t}$. An elastic-plastic model is introduced, and the following local constitutive incremental relations can be inferred:

$$
\begin{aligned}
& \delta \hat{F}_{n}=k_{n} \delta \hat{u}_{n} \\
& \delta \hat{\vec{F}}_{t}=\xi \frac{\hat{\vec{F}}_{t}+k_{t} \delta \hat{\vec{u}}_{t}}{\left\|\hat{\vec{F}}_{t}+k_{t} \delta \hat{\vec{u}}_{t}\right\|}-\hat{\vec{F}}_{t}
\end{aligned}
$$

where $\xi=\min \left\{\left\|\hat{\vec{F}}_{t}+k_{t} \delta \hat{\vec{u}} t\right\|, \tan \phi_{g}\left(\hat{\vec{F}}_{n}+k_{n} \delta \hat{\vec{u}}_{n}\right)\right\}, \quad k_{n} \quad$ is the normal elastic stiffness, $k_{t}$ is the tangential elastic stiffness, and $\varphi_{g}$ is the local friction angle.

\subsubsection{Microstructural expression of the macroscopic second-order work}

Starting from equation [11.7], and noting that the density of contact $\omega$ along each direction is expressed as $\omega=\frac{\omega_{e}}{V}$, where $\omega_{e}$ is the number of contacts along 
the considered direction, it follows that the differentiation of the Cauchy stress tensor is the sum of three terms:

$$
\delta \sigma_{i j}=\frac{2 r_{g}}{V} \iint_{D} \delta \hat{F}_{i} n_{j} \omega_{e} d \Omega+\frac{2 r_{g}}{V} \iint_{D} \hat{F}_{i} n_{j} \delta \omega_{e} d \Omega-\frac{\delta V}{V} \sigma_{i j}
$$

which can also be written as:

$$
\delta \sigma_{i j}+\frac{\delta V}{V} \sigma_{i j}-\frac{2 r_{g}}{V} \iint_{D} \hat{F}_{i} n_{j} \delta \omega_{e} d \Omega=\frac{2 r_{g}}{V} \iint_{D} \delta \hat{F}_{i} n_{j} \omega_{e} d \Omega
$$

Now, taking advantage of the kinematical projection relation yields:

$$
\begin{array}{r}
V \delta \sigma_{i j} \delta \varepsilon_{i j}+\delta V \sigma_{i j} \delta \varepsilon_{i j}-2 r_{g} \iint_{D} \hat{F}_{i} \delta \varepsilon_{i j} n_{j} d \omega_{e} d \Omega= \\
\iint_{D} \delta \hat{F}_{i} \delta \hat{u}_{i} \omega_{e} d \Omega
\end{array}
$$

Equation [11.12] can interestingly be compared to equation [11.4]. The term $V \overline{\bar{\sigma}}: \overline{\bar{L}}^{2}(\delta t)^{2}$, which is shown to be related to the change in texture [NIC 07c], can be assimilated to the term $2 r_{g} \iint_{D} \hat{F}_{i} \delta \varepsilon_{i j} n_{j} d \omega_{e} d \Omega$ which also accounts for textural change. In these conditions, it can be established that the macroscopic second-order work can be expressed in a very straightforward manner with respect to microscopic variables:

$$
W_{2}=\iint_{D} \delta \hat{F}_{i} \delta \hat{u}_{i} \omega_{e} d \Omega
$$

Coming back to a discrete formulation, integral $\iint_{D} \delta \hat{F}_{i} \delta \hat{u}_{i} \omega_{e} d \Omega$ corresponds to the summation of scalar product $\delta F_{i}^{c} \delta u_{i}^{c}$ over all the contacts contained within the assembly. As demonstrated by Nicot and Darve ([NIC 07a and c]), the term $\delta F_{i}^{c} \delta u_{i}^{c}$ can be interpreted as the microscopic second-order work associated with the contact " $c$ " between two given adjoining particles. As a consequence, equation 
[11.13] states that the macroscopic second-order work is equal to the sum of the microscopic second-order works associated with all the contacts existing within the assembly.

This basic result was inferred by considering a given constitutive relation, i.e. the micro-directional model. The purpose of the next section consists of generalizing this result without referring to any constitutive model.

\subsubsection{From micro to macro second-order work}

Let us consider a granular assembly containing $N$ grains " $p$ ", with $1 \leq p \leq N$. Each grain " $p$ " is in contact with $n_{p}$ other adjoining grains " $q$ ", with $1 \leq q \leq N$. Boundary particles $(p \in \partial V)$ are subjected to an external force $\vec{F}^{\text {ext,p }}$ directed by the external medium. We introduce the Galilean frame $\mathfrak{R}$, together with the local frame $\hat{R}\left\{\vec{n}, \vec{t}_{1}, \vec{t}_{2}\right\}$ attached to the considered contact whose normal to the tangent contact plane is $\vec{n} . \hat{\delta} \psi$ denotes the differentiation of any variable $\psi$ with respect to this frame.

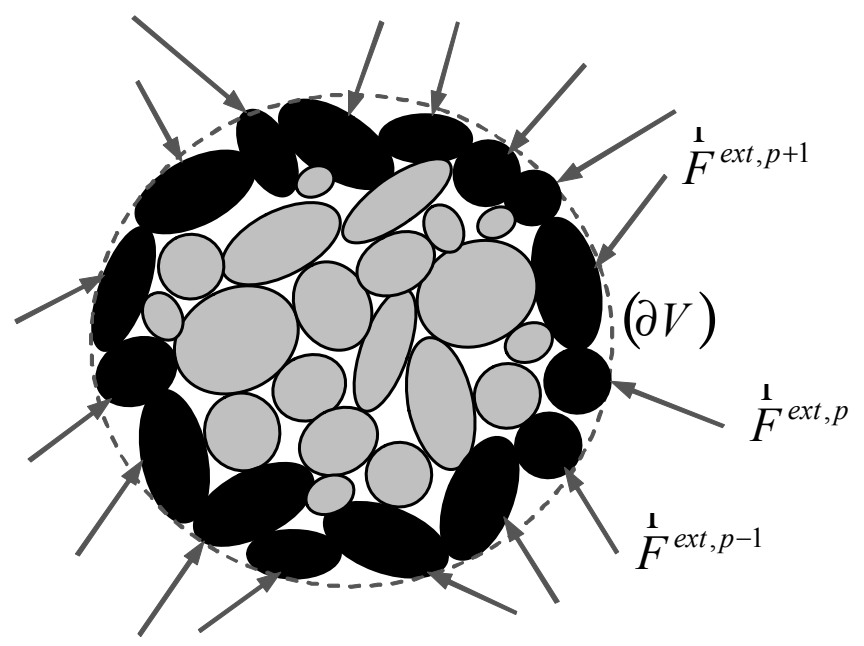

Figure 11.1. Granular assembly: boundary particles and external forces 

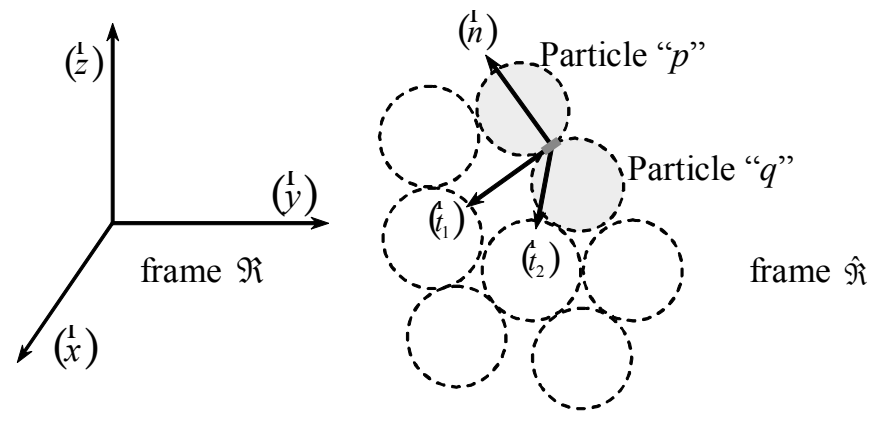

Figure 11.2. Galilean frame and local frame

The microscopic second-order work attached to the contact " $c$ " between particles " $p$ " and " $q$ " is given by the relation ([NIC 07a and c]):

$$
W_{2}^{p, q}=\hat{\delta} \vec{F}^{p, q} \cdot \hat{\delta}_{c}^{p, q}
$$

where $\hat{\delta} \vec{F}^{p, q}$ denotes the incremental contact force exerted by particle " $p$ " on particle " $q$ ", and $\hat{\delta} \vec{u}_{c}^{p, q}$ is the incremental relative displacement of particle " $p$ " with respect to particle " $q$ ".

On the granular assembly scale, the macroscopic second-order work can be related to the second-order time derivative of the kinetic energy as:

$$
W_{2}=\sum_{p \in \partial V} \delta \vec{F}^{e x t, p} \cdot \delta \vec{u}^{p}-\delta^{2} E_{c}(t)
$$

Taking into account the expression of the kinetic energy,

$$
\delta E_{c}(t)=\sum_{p=1}^{N}\left(\vec{F}^{p} \cdot \delta \vec{u}^{p}+\vec{M}^{p} \cdot \delta \vec{\omega}^{p}\right)
$$

we obtain, after some algebra ([NIC 07a]):

$$
W_{2}=\sum_{p=1}^{N} \sum_{q=1}^{p-1}\left(\hat{\delta} \vec{F}^{p, q} \cdot \hat{\delta}_{\hat{u}_{c}^{p}}^{p q}\right)-\sum_{p \in \partial V}\left(\vec{F}^{e x t, p} \cdot \delta^{2} \vec{u}^{p}\right)
$$


This basic relation indicates that the macroscopic second-order work is the sum of the microscopic second-order works extended to all the contacts of the whole assembly, $\quad \bar{W}_{2}=\sum_{p=1}^{N} \sum_{q=1}^{p-1}\left(\hat{\delta} \vec{F}^{p, q} \cdot \hat{\delta} \vec{u}_{c}^{p, q}\right)$, minus a boundary complementary term $\sum_{p \in \partial V}\left(\vec{F}^{e x t, p} \cdot \delta^{2} \vec{u}^{p}\right)$. This last term seems to be negligible from simulations based on a discrete element method [SIB 06 and 07]. This relation, that relates the macroscopic second-order work to microstructural elements embedded in the term $\bar{W}_{2}$, provides insight into the microstructural origins of the vanishing of the secondorder work. The next section is concerned with examining this feature.

\subsubsection{Micromechanical analysis of the vanishing of the second-order work}

Let us consider the term $\bar{W}_{2}=\sum_{p=1}^{N} \sum_{q=1}^{p-1}\left(\hat{\delta} \vec{F}^{p, q} \cdot \delta \vec{u}_{c}^{p, q}\right)$. The vanishing of $\bar{W}_{2}$ requires that the quantities $\hat{\delta} \vec{F}^{p, q} \cdot \delta \vec{u}_{c}^{p, q}$ vanish for a certain number of contacts. However, $\delta \vec{u}_{c}^{p, q}$ and $\hat{\delta} \vec{F}^{p, q}$ are related through constitutive equations such as those given in [11.9]. Considering any contact " $c$ ", $\delta \vec{u}_{c}$ splits into a normal component $\delta u_{c}^{n}$ and a tangential component $\delta u_{c}^{t}$. When the contact behaves in the plastic regime, the microscopic second-order work $W_{2}^{c}$ is a quadratic form that can be positive or negative:

$$
W_{2}^{c}=k_{n}\left(\delta u_{c}^{n}\right)^{2}+\tan \varphi_{g} \cos \alpha k_{n} \delta u_{c}^{t} \delta u_{c}^{n}+k_{t} \sin ^{2} \alpha\left(\delta u_{c}^{t}\right)^{2}
$$

where $\alpha$ is the angle between both vectors $\vec{t}_{1}=\frac{\vec{F}_{c}^{t}}{\left\|\vec{F}_{c}^{t}\right\|}$ and $\delta \vec{u}_{c}^{t}$. For axisymmetric conditions, $\alpha=0$, and equation [11.18] yields:

$$
W_{2}^{c}=k_{n}\left(\delta u_{c}^{n}\right)^{2}+\tan \varphi_{g} k_{n} \delta u_{c}^{t} \delta u_{c}^{n}
$$

The vanishing of $W_{2}^{c}$ requires that both following conditions are fulfilled [NIC 06]; [NIC 07a]: $\delta u_{c}^{n} \leq 0$ (unloading along normal direction) and $\delta u_{c}^{t} \geq-\frac{\delta u_{c}^{n}}{\tan \phi_{g}}$ (the amplitude of the tangential displacement is sufficient for the contact to behave 
plastically). It is worth noting that the microscopic second-order work is always positive when the contact undergoes a normal compression.

As in the plastic regime, $\delta F_{c}^{t}=k_{n} \tan \varphi_{g} \delta u_{c}^{n}$, condition $\delta u_{c}^{n} \leq 0$ also means that both components $\delta F_{c}^{n}$ and $\delta F_{c}^{t}$ are negative. Locally, on the contact scale, the stress state descends the Coulomb line, as seen in Figure 11.3. This result can be regarded as the microstructural origin of the fact that the vanishing of the macroscopic second-order work is essentially observed within the third quadrant, corresponding to $\delta \sigma_{1}<0$ and $\delta \sigma_{3}<0$ (in the stress incremental space), as seen for instance in Figure 11.4 (in some cases, negative values of the second-order work can also be observed within the first quadrant [DAR 04]).

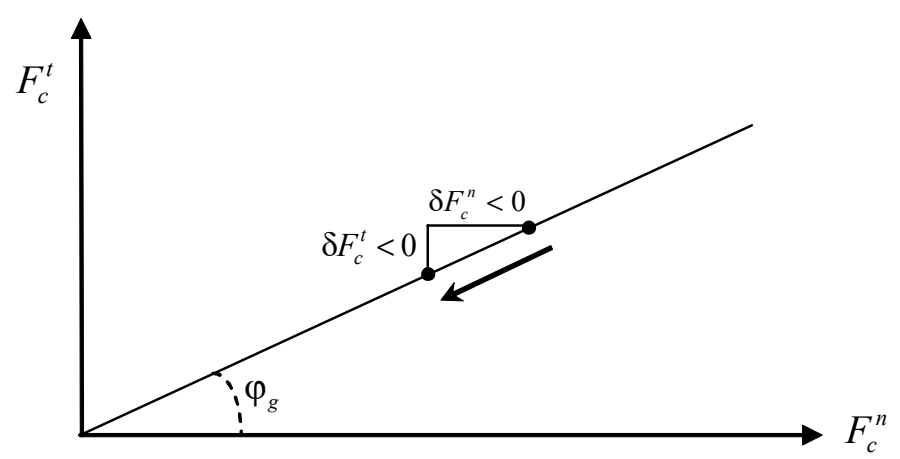

Figure 11.3. Evolution of the contact force for the vanishing of the microscopic second-order work: the contact force descends the Coulomb line
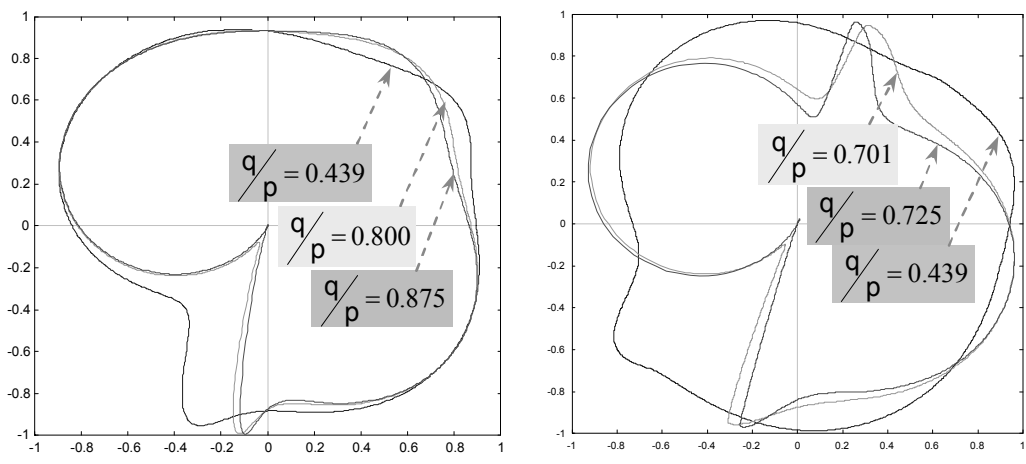

Figure 11.4. Polar representation of the second-order work along incremental stress direction (octo-linear model on the left side, micro-directional model on the right side) for different deviatoric ratios (after [NIC 06]) 


\subsection{Some remarks on the basic micro-macro relation for the second-order work}

Let us come back to further discuss relation [11.17]. This relation was investigated from discrete element simulations. Considering a cubic granular specimen, at rest after an initial axisymmetric drained triaxial loading, a series of stress probes was imposed, along all the directions of the incremental stress space, and both quantities $W_{2}$ (macroscopic second-order work) and $\bar{W}_{2}$ (sum of the microscopic second-order works) were compared.

As seen in Figure 11.5, equation [11.17] is perfectly verified within the elastic tensorial zone (the zone gathering loading directions leading to no plastic dissipation.), when contacts behave essentially elastically, and in a part of the plastic tensorial zone where plastic dissipation is related to sliding of contacts (Figure 11.6). As soon as loading directions are characterized by contact opening and/or creation (which corresponds to the central part of the plastic tensorial zone), a significant shift between $W_{2}$ and $\bar{W}_{2}$ exists (Figure 11.6). Should the validity of equation [11.17] be queried? It is our conviction that this basic relation is valid, irrespective of the tensorial zone considered. Nevertheless, it is worth noting that equation [11.17] applies to an equilibrium state; on the contrary, discrete element simulations require considering a finite time interval to calculate both quantities $W_{2}$ and $\bar{W}_{2}$. For loading directions belonging to the central part of the plastic tensorial zone (characterized by contact opening and creation), grain rearrangements continuously take place, so that the medium is (at least locally) no longer in equilibrium. As a consequence, for such loading directions, discrete element simulations do not constitute an appropriate way of checking a relation valid at the equilibrium but involving (force and displacement) rates.

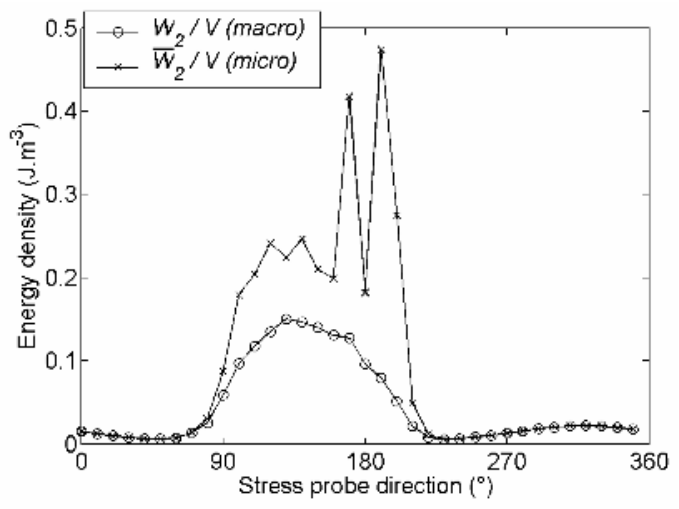

Figure 11.5. Microscopic and macroscopic second-order work density along different stress loading directions (after [NIC 07c]) 


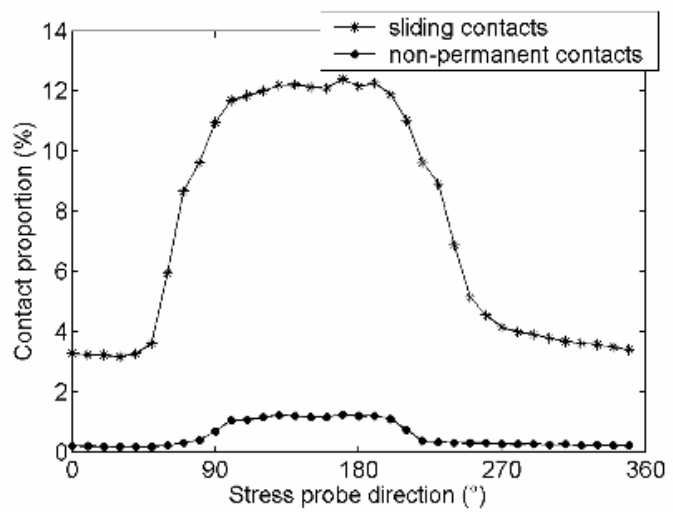

Figure 11.6. Grain rearrangement by sliding and opening/creation of contacts along different stress loading directions (after [DAR 07])

\subsection{Conclusion}

This chapter was devoted to the micromechanical investigation of the vanishing of the second-order work. As this quantity was shown to play a fundamental role in detecting the occurrence of a certain failure mode (diffuse failure mode, related to the spontaneous burst of kinetic energy), it is of great interest to understand what the microstructural conditions that lead to the vanishing of the second-order work are.

First, by considering our micro-directional model, it was inferred that the macroscopic second-order work of a given granular assembly is equal to the sum of the microscopic second-order works extended to all the contacts within the assembly. Then, the validity of the relation was extended based on general micromechanical arguments. This relation is fundamental since it bridges both microscopic and macroscopic worlds. The analysis was pursued by introducing an elastoplastic (frictional) model on the contact scale. The conditions for the vanishing of the microscopic second-order work (which is quadratic form with respect to the relative displacement) were examined, and an interpretation of the fact that the "unstable cones" containing the loading directions of the incremental stress space corresponding to negative values of $W_{2}$ are contained in the third quadrant ( $\delta \sigma_{1}<0$ and $\left.\delta \sigma_{3}<0\right)$ was provided. The microstructural ingredient of the analysis is essentially related to the local sliding condition. An important aspect remains to be considered in relation with the sudden (and brutal) deletion of contacts on the (mesoscopic) force chain scale. This geometrical aspect should be considered in addition to the former material aspect (sliding condition) considered in this chapter. 


\subsection{Bibliography}

[CHR 81] Christoffersen J., Mehrabadi M.M., Nemat-Nasser S., "A micro-mechanical description of granular material behavior", Journal of Applied Mechanics, vol. 48, pp. 339-344, 1981.

[DAR 04] Darve F., Servant G., Laouafa F., Khoa H.D.V., "Failure in geomaterials, continuous and discrete analyses", Comp. Methods Appl. Mech. Engrg., vol. 193, pp. 3057-3085, 2004.

[DAR 07] Darve F., Sibille L., DaOuadji A., Nicot F., "Bifurcations in granular media, macro- and micro-mechanics", Compte-Rendus de l'Académie des Sciences - Mécanique, vol. 335, pp. 496-515, 2007.

[HIL 58] HILl R., "A general theory of uniqueness and stability in elastic-plastic solids", J. Mech. Phys. Solids, vol. 6, pp. 236-249, 1958.

[LOV 27] Love A.E.H., A Treatise of Mathematical Theory of Elasticity, Cambridge University Press, Cambridge, 1927.

[MEH 82] Mehrabadi M.M., Oda M., Nemat-NAsser S., "On statistical description of stress and fabric in granular materials", Int. J. Num. Anal. Meth. Geomech., vol. 6, pp. 95-108, 1982.

[NIC 05] NiCOT F., DARVE F., "A multiscale approach to granular materials", Mechanics of Materials, vol. 37(9), pp. 980-1006, 2005.

[NIC 06] Nicot F., DARve F., "Micro-mechanical investigation of material instability in granular assemblies", Int. J. of Solids and Structures, vol. 43, pp. 3569-3595, 2006.

[NIC 07a] NiCOT F., DARVE F., "A micro-mechanical investigation of bifurcation in granular materials", Int. J. Solids and Structures, in press, 2007.

[NIC 07b] Nicot F., DARVE F., KHOA H.D.V., "Bifurcation and second-order work in geomaterials", Int. J. Num. Anal. Methods in Geomechanics, in press, 2007.

[NIC 07c] Nicot F., Sibille L., Donzé F., Darve F., "From microscopic to macroscopic second-order works in granular assemblies", Mechanics of Materials, vol. 39(7), pp. 664-684, 2007.

[SIB 06] SiBILle L., Modélisations discrètes de la rupture dans les milieux granulaires, $\mathrm{PhD}$ Thesis, Grenoble, INPG, 2006.

[SIB 07] Sibille L., NiCOt F., DonzÉ F., DARve F., "Material instability in granular assemblies from fundamentally different models", Int. J. Num. Anal. Methods in Geomechanics, vol. 31, pp. 457-481, 2007.

[WEB 66] WEBER J., "Recherches concernant les contraintes intergranulaires dans les milieux pulvérulents", Bulletin de Liaison des Ponts et Chaussées, no. 20, pp. 1-20, 1966. 